\title{
Effect of pelvic floor muscles exercise by extracorporeal magnetic innervations on the bladder neck and urinary symptoms: a research article
}

Cheng Yu Long ( $\sim$ urolong@yahoo.com.tw )

Kaohsiung Medical University Chung-Ho Memorial Hospital

Chieh Yu Chang

Kaohsiung Medical University Chung-Ho Memorial Hospital

Ching Hu Wu

Kaohsiung Medical University Chung-Ho Memorial Hospital

Kun Ling Lin

Kaohsiung Medical University Chung-Ho Memorial Hospital

Yi Yin Liu

Kaohsiung Municipal Hsiao-Kang Hospital

Zi Xi Lu

Kaohsiung Municipal Ta-Tung Hospital

\section{Research Article}

Keywords: stress urinary incontinence, extracorporeal magnetic innervation, bladder neck mobility, transperineal ultrasound, high-intensity focused electromagnetic muscle stimulation, pelvic floor muscle exercise

Posted Date: February 22nd, 2022

DOI: https://doi.org/10.21203/rs.3.rs-1214239/v1

License: (c) (1) This work is licensed under a Creative Commons Attribution 4.0 International License. Read Full License 


\section{Abstract}

Background: We aim to investigate the changes of the bladder neck (BN) and urinary symptoms before and after passive pelvic floor exercise by extracorporeal magnetic innervations (ExMl) therapy.

Methods: We assessed the BN and urinary symptoms of 20 women with stress urinary incontinence (SUI) by trans-perineal ultrasound and questionnaires before and after the ExMI therapy from January 2011 to February 2021.

Results: The urinary frequency and SUI incidences were significantly decreased following the ExMI therapy (McNemar's test; $P<0.01$ ). The therapeutic efficacy of SUI was $75 \%$. A significant decrease was noted in the pad test (paired t-test, $\mathrm{P}<0.05$ ). Nocturia had been improved in a marginally significance (McNemar's test, $\mathrm{P}=0.063$ ). Any difference in the pre- versus post-treatment regarding other urinary symptoms was not statistically significant. There was a significant difference in Urinary Distress Inventory (UDI-6) (paired t-test, $\mathrm{P}<0.001$ ), yet Incontinence Impact Questionnaire (IIQ-7) showed marginally significant difference (paired t-test, $P=0.066$ ). Three domains of lubrication, orgasm and satisfaction in the female sexual function index (FSFI) showed significant differences (paired t-test, $P$ $<0.05$ ), while other sexual symptoms had no significant difference before and after ExMI. Trans-perineal ultrasound found that bladder neck mobility and Q-tip straining angle were not statistically significant (paired t-test, $P>0.05$ ).

Conclusion: The ExMI is effective for SUI by strengthening the pelvic floor muscle with treatment efficacy of $75 \%$. However, it has a limited effect on bladder neck mobility, as evidenced by trans-perineal ultrasonography.

\section{Introduction}

Stress urinary incontinence (SUI) is a critical healthcare problem that can impact those who are affected socially, sexually, mentally, and financially. The principal mechanism of SUI is the weakening of pelvic floor support, which eventually leads to urethral hyper-mobility, while the minor mechanism is intrinsic sphincter deficiency. Any damage to the pelvic floor's nerves, muscles, and connective tissue can weaken the pelvic floor. Injury during childbirth, aging, menopausal status, and activities or medical conditions causing long-term increasing intra-abdominal pressure are all contributory factors [1], which explains why $\mathrm{SUl}$ is most common in older women.

Patients, especially those with less severe symptoms, usually opt for nonsurgical treatment initially. With the advancement of technology, several less invasive novel treatments have sprung up and shown promising potential. Electrical stimulation to improve pelvic floor function was developed by Caldwell et al. in 1963. The electrical current is emitted directly to excite the pelvic nerves and muscle, creating a muscle response similar to the Kegel exercise. Other mechanisms have also involved the reorganization of spinal reflex and regulation of cortical activity, which is suggested to be effective for frequency, urgency, and urge of incontinence [2]. 
However, in the clinical setting, the electrical stimulation requires the insertion of a probe into the vagina, which is considered inconvenient and less welcomed by female patients. The electric current needs to pass through the skin, subcutaneous tissues, and bone which causes high impedance and leads to higher current emitted through local tissue. Pain and discomfort such as local skin irritation are sometimes reported.

Extracorporeal magnetic innervation (ExMI) was developed by Calloway et al. in 1998 as the alternative to electrical stimulation and approved by the U.S. Food and Drug Administration in 2000. The electrified coils can induce an eddying current and produce a pulsed magnetic field that depolarizes the motor nerves and provokes muscle contraction [3,4]. Similarly, though the exact mechanism remains unclear, Galloway et al. claimed that ExMI treatment is also effective for frequency, urgency, urge, and mixed incontinence [4]. The magnetic field can penetrate all body tissues without significant alteration and passes uninterrupted through clothes $[3,4]$. Hence, without the need for intra-vaginal probe and undressing, ExMl is more acceptable by patients, providing a more comfortable and convenient treatment experience.

Most of the current literature has found ExMI treatment to be promising and effective, but objective measures are relatively inadequate; accordingly, the present prospective study aimed to investigate the exact change in the mobility and position of the bladder neck and urinary symptoms before and after ExMI therapy. Questionnaires, 1-hour pad test, and trans-perineal ultrasound were all used for both objective and subjective improvement assessment.

\section{Materials And Methods}

This study enrolled 20 female patients from January 2011 to February 2021 with demonstrable SUI, and all received the same ExMl treatment sessions, which took 20 minutes, twice a week, for 12 weeks. A complete evaluation was carried out before the treatment and, once again, six months following treatment. The evaluations included a 1-hour pad test, trans-perineal ultrasound, and a personal interview to complete the Urogenital Distress Inventory (UDI-6), the Incontinence Impact Questionnaire (IIQ-7) [5], the Female Sexual Function Index (FSFI) [6] questionnaires, and urinary symptoms with the standardized questionnaire taking into account the 2002 ICS definitions [7].

The results of the ExMI treatment were assessed subjectively and objectively. "Cure" was defined as a patient who had no more SUI symptoms with urine loss of less than $2 \mathrm{~g}$ on pad test. "Improvement" was defined as urine loss of $2-5 \mathrm{~g}$ on pad test. Leakage of more than $5 \mathrm{~g}$ on pad test was deemed a "failure" [8].

The bladder neck's anatomical position and dynamic mobility were evaluated by trans-perineal ultrasound with the patient in the supine position. The curved linear-array transducer was placed sagittally and visualized the symphysis pubis, vesicourethral junction, urethra, and bladder. The bladder neck position was measured in the $x-y$ coordinate system with the inferior edge of the pubic bone as the reference point (Figure 1). Patients were ordered to perform a Valsalva maneuver to record the point of maximal bladder neck descent. 
The Ethics Committee of Kaohsiung University and Teaching Hospital approved the study protocol. A statistical analysis of the data was calculated using paired t-test for parametric continuous variables, the McNemar's test for categorical variables and the Fisher's exact test for small sample sizes. A difference was considered statistically significant when $P$ value $<0.05$.

\section{Results}

The average age of all participants is 46.8 ; the mean parity is 2.2 . The mean BMI was $23.8 \mathrm{~kg} / \mathrm{m}^{2}$. Two patients were postmenopausal, and one patient was under hormone replacement therapy before initiating the therapy and during the follow-up months. One patient had diabetes mellitus, and eight had hypertension under medical control (Table. 1). All the patients were neurologically ordinary and nonpregnant. None of the patients had pelvic organ prolapse of more than stage 1 (i.e., any most distal portion of prolapse was one centimeter or more above the level of vaginal introitus).

In the present study, with 20 patients enrolled in the 12 weeks of ExMl therapy, urinary frequency, SUI, and nocturia were the most common pre-treatment symptoms. The urinary frequency and SUI incidences were significantly decreased following the ExMl therapy (McNemar's test; $\mathrm{P}<0.05$ ). The therapeutic effect of SUI was $75 \%$, with the cure group accounting for $50 \%$ and the improvement group for $25 \%$. Nocturia was improved in five patients, which presented as marginally significant (McNemar's test; P value slightly $>0.05$ ). Profound decrease with statistical significance was noticed in the pad test (paired t-test, $P<0.05$ ). Any difference in the pre- versus the post-therapy incidence of other urinary symptoms was not statistically significant. There was a significant difference in UDI-6, but in IIQ-7, the result showed marginally significant (paired t-test, $\mathrm{P}=0.066)($ Table 2).

As for the female sexual function, significant differences over three domains: lubrication, orgasm, and satisfaction were noted after the ExMl therapy (paired t-test, $P<0.05$ ). Sexual desires and sexual arousal had no significant difference before and after the therapy. Dyspareunia had no significant improvement as well. (Table 3 ).

Trans-perineal ultrasound was performed pre- and post-therapy to evaluate the bladder neck mobility at rest and during straining. The topography of the bladder neck showed no significant difference between the pre- and the post-therapy, either at rest or during straining (paired t-test, $P>0.05$ ). The bladder neck mobility also showed no statistically significant difference (paired t-test, $P>0.05$ ) (Figure 2 ). In conclusion, from the trans-perineal ultrasound, we noticed no significant difference over the bladder neck hypermobility. As for the Q-tip straining angle, there was also no statistical difference (paired t-test, $\mathrm{P}$ $>0.05$ ) (Table 4).

\section{Table 1}

Demographic data $(n=20)$ are given as mean \pm standard deviation or $n(\%)$ 


\begin{tabular}{|l|l|}
\hline Mean age (years) & $46.8 \pm 11.9$ \\
\hline Mean parity & $2.2 \pm 1.2$ \\
\hline Mean body mass index (BMI) $\left(\mathrm{kg} / \mathrm{m}^{2}\right)$ & $23.8 \pm 3.3$ \\
\hline Menopause & $2(10)$ \\
\hline Current hormonal therapy & $1(4)$ \\
\hline Diabetes mellitus & $1(4)$ \\
\hline Hypertension & $8(32)$ \\
\hline Follow-up months & 3 \\
\hline
\end{tabular}

Table 2

Urinary symptoms and pad tests before and 6 months after ExMI. Data are given as $n(\%)$ or mean \pm standard deviation 


\begin{tabular}{|c|c|c|c|}
\hline Symptoms & Pre-ExMI $(n=20)$ & Post-ExMI $(n=20)$ & P-value \\
\hline Urinary frequency & $12(60)$ & $2(10)$ & $0.002 * \S$ \\
\hline Stress urinary incontinence & $20(100)$ & $15(75)^{\wedge}$ & $<0.001 \star \S$ \\
\hline Cure & & $10(50)$ & \\
\hline Improvement & & $5(25)$ & \\
\hline Failure & & $5(25)$ & \\
\hline Urgency urinary incontinence & $7(35)$ & $2(10)$ & $0.11+$ \\
\hline Feeling of incomplete emptying & $1(5)$ & $0(0)$ & $0.9+$ \\
\hline Hesitancy & $1(5)$ & $0(0)$ & $0.9+$ \\
\hline Nocturia & $8(40)$ & $3(15)$ & $0.063^{\star}$ \\
\hline Pad test (g) & 307 & 25 & $0.046 \# \S$ \\
\hline UDI-6 & $44.4 \pm 14.4$ & $24.1 \pm 16.6$ & $<0.001 \# \S$ \\
\hline QII-7 & $43.6 \pm 41.3$ & $21.2 \pm 23.7$ & 0.066 \\
\hline \multicolumn{4}{|c|}{ ExMl, extracorporeal magnetic innervation; } \\
\hline \multicolumn{4}{|c|}{ * McNemar's test, +Fisher's exact test, \# Paired $t$-test. } \\
\hline \multicolumn{4}{|c|}{$\begin{array}{l}\text { § Statistical significance } \\
\text { } \text { SUl post-ExMl treatment was categorized into three groups. Cure \& improvement group (total n=15) } \\
\text { showed statistical significance in ExMl treatment). }\end{array}$} \\
\hline
\end{tabular}

\section{Table 3}

Changes in scores of female sexual function index before and 3 months after ExMI. Data are given as mean \pm standard deviation. 


\begin{tabular}{|llll|}
\hline Domains & Pre-ExMI $(\mathrm{n}=20)$ & Post-ExMI $(\mathrm{n}=20)$ & P-value \\
\hline Sexual desire & $2.8 \pm 0.5$ & $2.6 \pm 0.8$ & 0.77 \\
\hline Sexual arousal & $2.9 \pm 0.9$ & $3.4 \pm 0.6$ & 0.48 \\
\hline Lubrication & $4.1 \pm 0.8$ & $4.7 \pm 1.3$ & $0.027 \star \S$ \\
\hline Orgasm & $3.4 \pm 0.9$ & $4.1 \pm 0.5$ & $0.012 \star \S$ \\
\hline Satisfaction & $3.7 \pm 0.9$ & $4.6 \pm 0.8$ & $0.016 \star \S$ \\
\hline Dyspareunia & $4.6 \pm 1.3$ & $4.9 \pm 1.3$ & 0.18 \\
\hline Total scores & $21.5 \pm 4.8$ & $24.3 \pm 5.6$ & 0.69 \\
\hline ExMl, extracorporeal magnetic innervation; & \\
* Paired $t$-test; $§$ statistical significance. & \\
\hline
\end{tabular}

Table 4

Topography of the bladder neck (BN) at rest and during straining, and its mobility assessed by perineal ultrasonography in pre- and postoperative evaluation. Values area given as mean \pm standard deviation $(\mathrm{mm})$ or $\mathrm{n}(\%)$.

\begin{tabular}{|llllllll|}
\hline $\begin{array}{l}\text { ExMl } \\
\text { group } \\
(\mathrm{n}=20)\end{array}$ & Rest & & Straining & & $\begin{array}{l}\text { Mobility } \\
(\mathbf{m m})\end{array}$ & $\begin{array}{l}\text { BN } \\
\text { hypermobility }\end{array}$ & $\begin{array}{l}\text { Q-tip } \\
\text { straining } \\
\text { angle ( })\end{array}$ \\
\cline { 2 - 8 } & Dx & Dy & Dx & Dy & & & 41.1 \\
\hline Pre-ExMI & $-4.2 \pm 6.1$ & $21.2 \pm 2.2$ & $-11.8 \pm 6.9$ & $9.2 \pm 10.9$ & $10.9 \pm 4.7$ & $9(56.3)$ & 44.4 \\
\hline Post-ExMl & $-2.7 \pm 5.1$ & $21.8 \pm 3.9$ & $-12.6 \pm 5.3$ & $9.7 \pm 9.9$ & $11.8 \pm 6.4$ & $11(68.8)$ & 0.57 \\
\hline P-value & 0.24 & 0.49 & 0.69 & 0.86 & 0.42 & 0.5 & \\
\hline
\end{tabular}

ExMI, Extracorporeal magnetic innervation; * Paired t-test; § statistical significance.

\section{Discussion}

The importance of pelvic floor muscle (Kegel's) exercise in treating women with SUl is beyond description, although requiring proper technique and commitment to performing for a long time to have a better outcome [9]. For women who cannot identify the proper muscle or regularly conduct the exercise, treatments such as electric stimulation and ExMI can be introduced. They both shared the same goal: to strengthen the pelvic floor muscles passively. In the clinical situation, the strong point of the ExMI is convenience and comfort compared to electrical stimulation. 
In our observation, most of the current studies that discussed the therapeutic effect of ExMI lack objective measurements. As a result, 1-hour pad test and trans-perineal ultrasound were then performed explicitly in this study. Trans-perineal ultrasound is a cheap, real-time, and non-invasive method to evaluate genitourinary displacement [10].

In the present study, the incidence of SUI decreased significantly following the treatment. The pad test also showed considerable improvement. The therapeutic effect of SUI was calculated to be $75 \%$. The topography of the bladder neck collected from trans-perineal ultrasound showed no significant improvement over bladder neck hypermobility. These results are reasonable with the primary mechanism of the ExMl, which helps the pelvic floor muscle strengthen by contracting passively. Even though the hypermobility of the bladder neck remains, the stronger pelvic floor muscle can help with the closure of the urethra and compensate for the descent of the bladder neck at straining.

Urinary symptoms related to the detrusor muscle and pelvic plexus nerve systems such as urgency incontinence, incomplete emptying, and hesitancy showed no significant difference after treatment in the study; however, the incidence of frequency had significantly decreased following treatment. The complicated mechanism of electrical stimulation and ExMl treating detrusor overactivity involves stimulating the afferent pudendal nerve or sacral root to inhibit the pelvic efferent (parasympathetic) or activate the hypogastric efferent (sympathetic) [11].

ExMI penetrates the body tissue, and the magnitude of the field only falls off as the inverse square of the distance, which helps activate deeper proximal nerves that are difficult to reach by electrical stimulation without hurting the skin. Tomonori et al. reported in a review article that for urgency incontinence and mixed urinary incontinence, neuromodulation could be the treatment alternative to drug therapy because of its efficacy and much fewer side effects [12].

Lubrication, orgasm, and satisfaction had significantly improved following the therapy, although sexual arousal and desire showed no significant difference. The fear of coital incontinence can be a source of frustration, anxiety, and low self-esteem to women with SUI [13]; eventually, sexual functioning can be affected. Bekker et al. in 2009 mentioned that the improvement in coital incontinence resulted in improvement of sexual function. The present study found similar results where ExMI could significantly improve the pelvic floor muscle and improve female sexual function. It is reasonable to postulate that women would appear to be more willing to relax and enjoy sexual activity when urinary incontinence resolves; on that account, sexual arousal and desire might be improved.

As a result, ExMI can effectively improve SUI by providing pelvic floor muscle stimulation in a painless and convenient (no need for undressing) way. The ability of sexual function change is secondary to the improvement of pelvic floor muscle power. R Lim et al., who performed a one-year follow-up, found high patient acceptance and low dropout rates [14]. This can be interpreted as ExMI being an attractive and promising non-surgical alternative for patients who do not want surgery. 
HIFEM (High-Intensity Focused Electromagnetic) is a novel technology that offers supramaximal pelvic floor contraction using focused electromagnetic energy. The supramaximal pelvic floor contraction is brain-independent and beyond physiological contraction in strength and repetitiveness, leading to pelvic floor stimulation, adaptation, and remodeling [15]. HIFEM is conducted with the patient fully clothed and seated. As mentioned in several studies, urinary incontinence and sexual function had significantly improved after HIFEM treatment [16].

It is worth noting that trans-perineal ultrasound was widely used for its real-time convenience and objectiveness in many recent studies we reviewed. The diameter of the anteroposterior and latero-lateral levator hiatus and hiatal area (HA) were significantly reduced in the group treated with HIFEM technology compared with the group treated with the electrical device [17].

The FDA (U.S. food and drug administration) approved the indication of the noninvasive Er: YAG laser in the field of urogynecology in 2014. The Er: YAG vaginal laser produces a non-ablative thermal effect that causes the remodeling of vaginal connective tissue and hence, provides the vaginal wall with strength and mechanical traction [18]. The benefit of remodeling can extend to the lower urinary tract anatomy and further improve SUI. Lin KL and Long CY et al. found a significant decrease in the bladder neck mobility via perineal ultrasonography [19]. The mechanism of vaginal laser in treating SUI should be different from ExMl, as it mainly focuses on neocollagenesis and remodeling of connective tissue.

There are some limitations to our study. Firstly, a relatively small number of participants were enrolled; and secondly, a longer follow-up to determine how long the benefits of treatment will be sustained was lacking. A randomized controlled trial including a control group and a larger sample size is necessary to support our findings. However, the strength of our study was applying objective trans-perineal ultrasound measurement instead of subjective questionnaires only.

\section{Conclusions}

The ExMI effectively treats SUI by strengthening the pelvic floor muscle painlessly and conveniently without a significant decrease in bladder neck mobility found by trans-perineal ultrasonography. The treatment mechanism of ExMl should be very different from vaginal laser and surgery. Whether HIFEM provides a more substantial or more extended effect remains exploratory. More randomized controlled studies and longer follow-up are expected to confirm and elucidate our findings.

\section{Abbreviations}

SUI: Stress urinary incontinence

BN: Bladder neck

ExMl: Extracorporeal magnetic innervation

UDI-6: Urogenital Distress Inventory 
IIQ-7: Incontinence Impact Questionnaire

FSFI: Female Sexual Function Index

HIFEM: High-Intensity Focused Electromagnetic

\section{Declarations}

\section{Ethics approval and consent to participate:}

all experimental protocols were approved by the Ethics Committee of Kaohsiung University and Teaching Hospital. All methods were carried out in accordance with relevant guidelines and regulations. Informed consent was obtained from all subjects and/or their legal guardian(s).

\section{Consent for publication:}

not applicable

\section{Availability of data and materials:}

The datasets used and analysed during the current study are available from the corresponding author on reasonable request.

\section{Competing interests:}

not applicable

\section{Funding:}

not applicable

\section{Authors' contributions:}

$\mathrm{CY}$ Chang and $\mathrm{CH}$ Wu were major contributors in writing the manuscript. KL Lin, YY Liu, ZX Loo collected and analyzed the patient data. CY Long conceived and designed the analysis. All authors read and approved the final manuscript."

\section{Acknowledgements:}

not applicable 


\section{References}

1. Norton, P. A. Prevalence and social impact of urinary incontinence in women. Clin Obstet Gynecol 1990, 33(2): 295-297. doi: 10.1097/00003081-199006000-00013.

2. Schreiner, L., et al. Electrical stimulation for urinary incontinence in women: a systematic review. Int Braz J Urol 2013, 39(4): 454-464. doi: 10.1590/S1677-5538.IBJU.2013.04.02.

3. Galloway, N. T., et al. Extracorporeal magnetic innervation therapy for stress urinary incontinence. Urology 1999, 53(6): 1108-1111. doi: 10.1016/s0090-4295(99)00037-0.

4. Galloway, N. T., et al. Update on extracorporeal magnetic innervation (EXMI) therapy for stress urinary incontinence. Urology 2000, 56(6 Suppl 1): 82-86. doi: 10.1016/s0090-4295(00)00686-5.

5. Uebersax JS, et al. Short forms to assess life quality and symptom distress for urinary incontinence in women: the Incontinence Impact Questionnaire and the Urogenital Distress Inventory. Continence Program for Women Research Group. Neurourol Urodyn. 1995, 14(2):131-9. doi: 10.1002/nau.1930140206.

6. Rosen R, et al. The Female Sexual Function Index (FSFI): a multidimensional self-report instrument for the assessment of female sexual function. J Sex Marital Ther. 2000, 26(2):191208. https://doi.org/10.1080/009262300278597

7. Abrams P, et al. Standardisation Sub-committee of the International Continence Society. The standardisation of terminology of lower urinary tract function: report from the Standardisation Subcommittee of the International Continence Society. Neurourol Urodyn. 2002;21(2):167-78. doi: 10.1002/nau.10052.

8. Long, C. Y., et al. Clinical and ultrasonographic comparison of tension-free vaginal tape and transobturator tape procedure for the treatment of stress urinary incontinence. J Minim Invasive Gynecol 2008, 15(4): 425-430. doi: 10.1016/j.jmig.2008.03.003.

9. Culbertson, S. and A. M. Davis. Nonsurgical Management of Urinary Incontinence in Women. Jama 2017, 317(1): 79-80. doi:10.1001/jama.2016.18433

10. Yamanishi, T., et al. Randomized, double-blind study of electrical stimulation for urinary incontinence due to detrusor overactivity. Urology 2000, 55(3):353-357. doi: 10.1016/s0090-4295(99)00476-8.

11. Yamanishi, T., et al. Neuromodulation for the treatment of urinary incontinence. Int J Uro/ 2008, 15(8): 665-672. doi: 10.1111/j.1442-2042.2008.02080.x

12. Long, C. Y., et al. Effect of laparoscopic hysterectomy on bladder neck and urinary symptoms. Aust $N$ Z J Obstet Gynaecol 2003, 43(1): 65-69. doi:10.1046/j.0004-8666.2003.00019.x

13. Fatton, B., et al. Stress urinary incontinence and LUTS in women-effects on sexual function. Nat Rev Uro/ 2014, 11(10): 565-578. doi: 10.1038/nrurol.2014.205.

14. Lim, R., et al. Responsiveness of the Golombok Rust Inventory of Sexual Satisfaction in couples with incontinent partners. Eur J Obstet Gynecol Reprod Bio/ 2018, 222: 109-

112. https://doi.org/10.1016/j.ejogrb.2018.01.024 
15. Silantyeva, E., et al. A Comparative Study on the Effects of High-Intensity Focused Electromagnetic Technology and Electrostimulation for the Treatment of Pelvic Floor Muscles and Urinary Incontinence in Parous Women: Analysis of Posttreatment Data. Female Pelvic Med Reconstr Surg 2021, 27(4): 269-273. doi:10.1097/SPV.0000000000000807.

16. Tc, H., et al. The Use of HIFEM Technology in the Treatment of Pelvic Floor Muscles as a Cause of Female Sexual Dysfunction: A Multi-Center Pilot Study. J Women's Health Care 2019, 8:1. DOI: 10.4172/2167-0420.1000455

17. Alinsod R., V. V., Yanev K, Buzhov B., Stoilov M., Yanev K., Georgiev M. HIFEM® TECHNOLOGY - A NEW PERSPECTIVEIN TREATMENT OF STRESS URINARY INCONTINENCE. 38th American Society for Laser Medicine and Surgery Annual Conference, 2018.

18. Gaspar, A., et al. Efficacy of Erbium:YAG laser treatment compared to topical estriol treatment for symptoms of genitourinary syndrome of menopause. Lasers Surg Med 2017, 49(2): 160-168. doi: 10.1002/lsm.22569.

19. Lin, K. L., et al. Effect of Er:YAG Laser for Women with Stress Urinary Incontinence. Biomed Res Int 2019: Article ID 7915813, 7 pages. https://doi.org/10.1155/2019/7915813

\section{Figures}

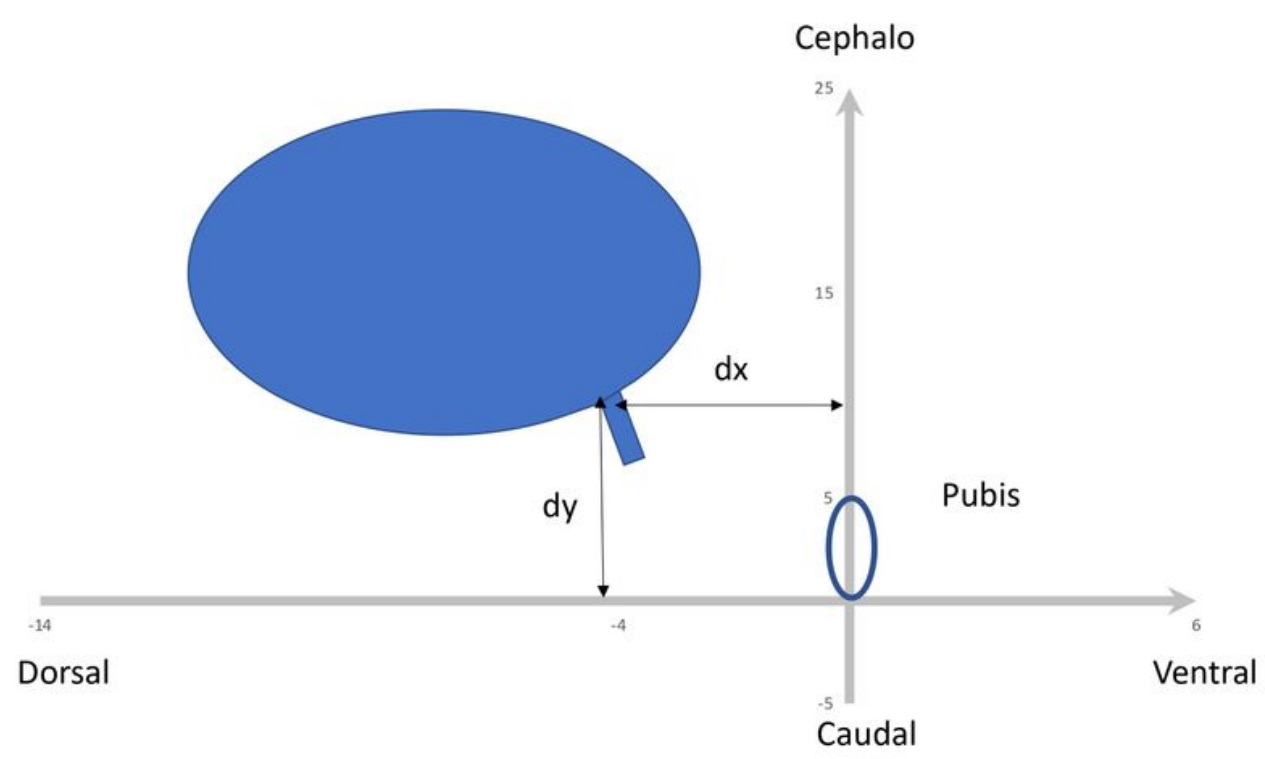

Figure 1 Evaluation of bladder neck location on introital ultrasonography relative to reference coordinate system. Dx, the distance between the bladder neck and the central line of the symphysis; Dy, the distance between the bladder neck and the axis perpendicular to the central line of the symphysis.

\section{Figure 1}




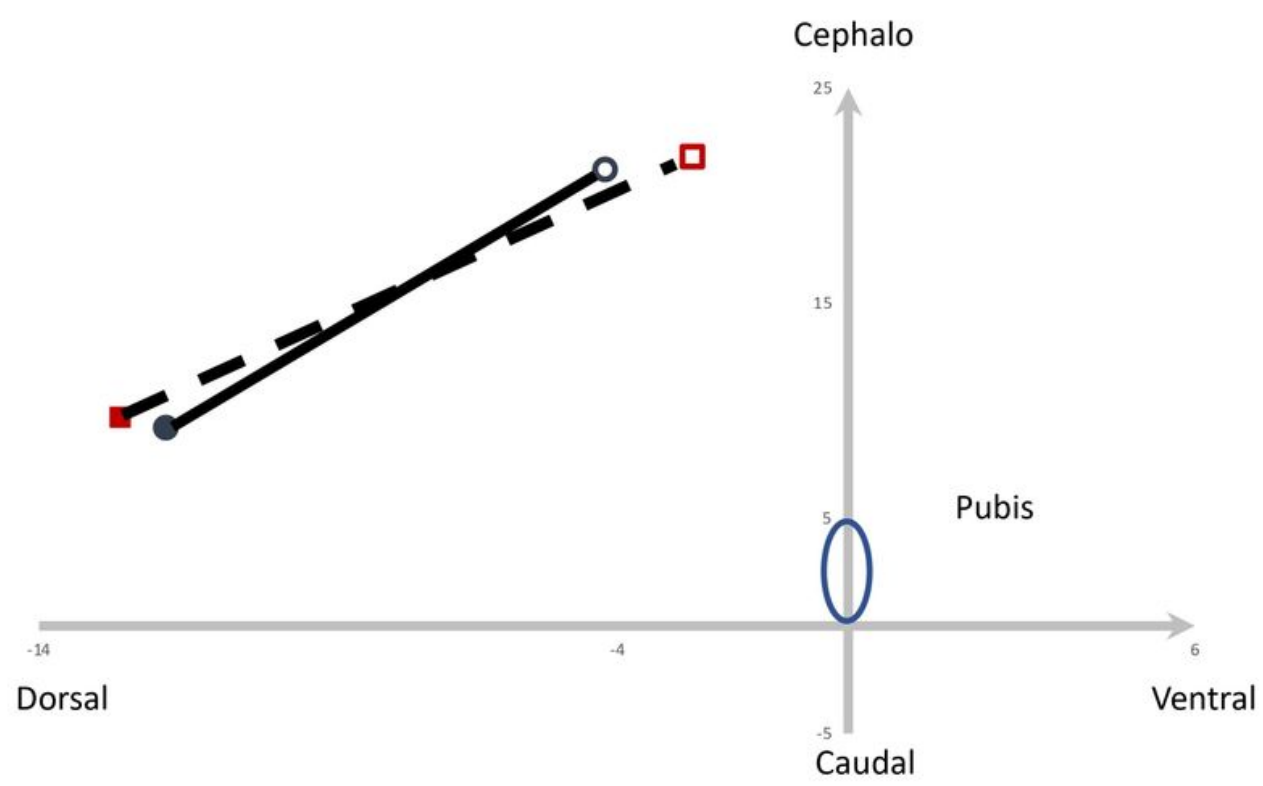

Figure 2 Bladder neck localisation of the patients before and after ExMI treatment. The bladder neck position is represented as:

before treatment: $\bigcirc$ is at rest; 1 is during straining; and straight line represented mobility; after treatment: $\square$ is at rest; $\mathbf{a}$ is during straining; and dotted line represented mobility.

\section{Figure 2}

Please See image above for figure legend. 